\title{
Grain Growth, Alignment, and Properties of Bi-based Superconducting Materials
}

\author{
R. Funahashi, I. Matsubara, K. Ueno, H. Ishikawa, and H. Yamashita \\ Osaka National Research Institute, AIST, Midorigaoka, Ikeda, Osaka 563, Japan
}

\begin{abstract}
Hot pressing effect on critical current density $\left(J_{c}\right)$ is studied on $\mathrm{Bi}_{2} \mathrm{Sr}_{2} \mathrm{CaCu}_{2} \mathrm{O}_{\mathrm{x}}(\mathrm{Bi}-2212)$ superconducting materials. Hot pressing is effective to hasten the grain growth, align the grains, and improve the bulk density of the $\mathrm{Bi}-2212$ materials. In consequence of hot pressing, $J_{c}$ increases by more than 10 times at $77 \mathrm{~K}$ under zero magnetic field. Hot pressing effect is enhanced by incorporation of large single crystalline Bi-2212 whiskers. $J_{c}$ is $3.1 \times 10^{3} \mathrm{~A} / \mathrm{cm}^{2}$, which is about 8 times higher than that for the hot pressed $\mathrm{Bi}-2212$ material without the whiskers, at $77 \mathrm{~K}$ under zero magnetic field for the $\mathrm{Bi}-2212$ whisker composite. This is due to the haste of grain growth and improvement of grain alignment around the whiskers.
\end{abstract}

[Bi-2212 superconducting materials, critical current density, hot pressing, microstructure, whisker composite]

\section{Introduction}

Oxide superconducting materials for electric power application are composed of sintered ceramics at present. Therefore, critical current density $\left(J_{c}\right)$, which is most important parameter for electric power application, is determined by properties of grain boundaries. That is to say, decrease in the number of grain boundaries through which the superconducting current flows and strengthening of superconducting coupling at the grain boundaries are effective for high $J_{c}$. Increase in grain size, and improvement of grain alignment and bulk density solve the problem of the grain boundaries. Many method for preparing these materials have been reported, for example, an Ag-sheathed method [1], a doctor-blade casting method [2], and a hot pressing method [3]. Especially, hot pressing is advantageous to prepare highly aligned and densified bulk samples with large grains.

On the other hand, the growth of $\mathrm{Bi}_{2} \mathrm{Sr}_{2} \mathrm{CaCu}_{2} \mathrm{O}_{\mathrm{x}}$ (Bi2212) whiskers of single crystals has been reported [4] and they have a ribbon-like shape with well grown $a b$-plane. Because of their ribbon-like shape, the whiskers can be easily aligned by pressing. Therefore, by incorporation of the whiskers into Bi-2212 polycrystalline ceramics, it is expected to obtain highly aligned $\mathrm{Bi}-2212$ superconducting whisker composites in which the $\mathrm{Bi}-2212$ matrix grains are grown well and aligned parallel to the whiskers by hot pressing. This paper presents the effect of hot pressing on microstructure; grain growth, grain alignment, and bulk density, and on superconducting properties of the Bi-2212 materials. Moreover, the effect of incorporation of the whiskers into the hot pressed $\mathrm{Bi}-2212$ materials on the microstructure and superconducting properties are also studied.

\section{Experimental}

In this study, a commercially available $\mathrm{Bi}-2212$ powder (DOWA, $3 \mathrm{~N}$, average particle size $<3 \mathrm{~mm}$ ) was used to prepare samples. No impurity phases included in this powder were checked by X-ray diffraction (XRD) measurement. This powder was pelletized under $54 \mathrm{MPa}$. After pelletization, a Bi2212 sintered sample was prepared under $1 \mathrm{~atm}$ at $870^{\circ} \mathrm{C}$ for $40 \mathrm{~h}$ and a Bi-2212 hot pressed sample was obtained by hot pressing under $12 \mathrm{MPa}$ at $815^{\circ} \mathrm{C}$ for $24 \mathrm{~h}$.

The Bi-2212 whiskers were prepared by heating meltquenched glass plates with the composition of $\mathrm{BiSrCaCu}_{2} \mathrm{Al}_{\mathrm{x}} \mathrm{O}_{\mathrm{y}}(0.5<\mathrm{x}<0.75)$ in a stream of $\mathrm{O}_{2}$ gas at $865^{\circ} \mathrm{C}$ for $80 \mathrm{~h}$. The powder $(4.50 \mathrm{~g})$ and the whiskers $(0.50 \mathrm{~g}, 10$ wt. \% composite) were mixed in hexane by using a magnetic stirrer for $10 \mathrm{~min}$. The mixture was dried and pelletized under $54 \mathrm{MPa}$. The pellet was hot pressed for $24 \mathrm{~h}$ at $815^{\circ} \mathrm{C}$ under 12 $\mathrm{MPa}$.

After preparation, all samples were cut to rods for measurements. Transport properties were measured by a standard four-probe method under zero magnetic field. XRD measurement and microstructural observation using a scanning electron microscope (SEM) were performed on the pressed plane and cross section.

\section{Results and discussion}

\section{3-1. Hot pressing effect}

Figs. 1 show SEM photographs for the cross section of the sintered sample (a) and the hot pressed sample (b). It is clear from these photographs that hot pressing is effective to hasten the grain growth and improve the grain alignment. Ratios of XRD peak intensity of $(00 \underline{10})$ to (200), a measure of grain alignment, for the pressed plane in the pelletization step and for the cross section are shown on Table 1. This result also indicates the improvement of grain alignment by hot pressing. Moreover, hot pressing makes bulk density high by more than 2 times. The microstructural improvement by hot pressing leads to increase in zero resistance temperature $\left(T_{c, z e r o}\right)$ and $J_{c}$. It is clear from the result of XRD that the $a b$-plane of the $\mathrm{Bi}-2212$ grains are aligned rather parallel to the pressed plane even in the 
sintered sample. This is due to the plate-like structure of the $\mathrm{Bi}-2212$ grains with well grown $a b$-plane. The Bi-2212 grains are aligned in the pelletization step. However, the pressing effect on the grain alignment is enhanced by hot pressing. Although the grain growth is progressed in random direction in sintering step under $1 \mathrm{~atm}$, it is restricted in a plane parallel to the pressed plane during hot pressing. This restriction of direction of the grain growth enhances the grain alignment in the hot pressed sample. Moreover, the grain growth is hastened by hot pressing. This seems to be due to increase in diffusion coefficient in the solid state by hot pressing.
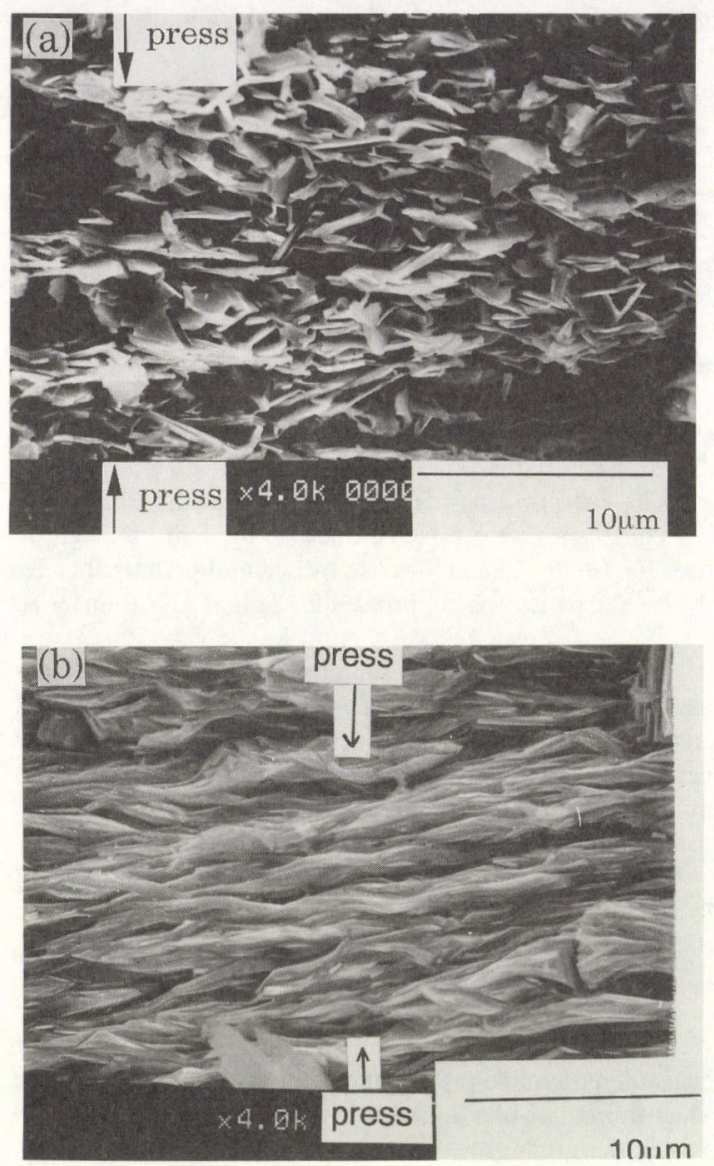

Fig. 1 SEM photographs for the cross section of the sintered sample (a) and the hot pressed sample (b).

\section{3-2. Effect of incorporation of Bi-2212 whiskers}

Fig. 2 is a SEM photograph for the cross section of the whisker composite. The stacking layered structure of the whiskers, which corresponds to the $a c$ or $b c$-plane, is observed. The whiskers have a ribbon-like shape with well grown $a b$ plane [4], so that their $a b$-plane are aligned parallel to the pressed plane by pressing in pelletization and hot pressing steps.

The haste of grain growth and the improvement of grain alignment between the whiskers is clear. In the hot pressed sample without the whiskers, pressure deviated from the uniaxial hot pressing direction is applied between the grains because of their small grain size, especially, it is remarkable in the farther from the surface, although the grains have the

Table 1. Hot pressing effects on the grain growth, alignment, bulk density, and superconducting properties.

\section{sintered sample hot pressed sample}

\begin{tabular}{lcc} 
XRD intensity ratio & & \\
$(00 \underline{10}) /(200)$ & & \\
pressed plane & 0.66 & 34 \\
cross section & 0.23 & 0.03 \\
Grain size & small & large \\
Bulk density $\left(\mathrm{g} / \mathrm{cm}^{3}\right)$ & 2.7 & 5.8 \\
$T_{c, z e r o}(\mathrm{~K})$ & 74 & 84 \\
$J_{c}\left(\mathrm{~A} / \mathrm{cm}^{2}\right)$ & $34($ at $69 \mathrm{~K})$ & $4.0 \times 10^{2}$ (at $\left.77 \mathrm{~K}\right)$ \\
\hline
\end{tabular}

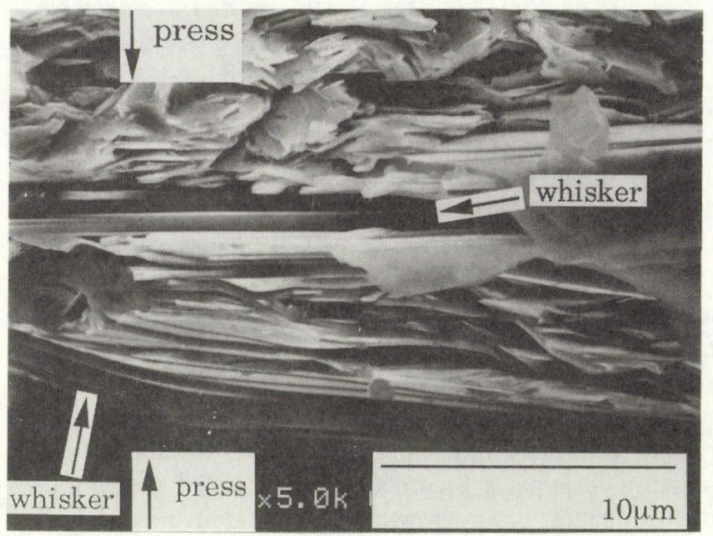

Fig. 2 SEM photograph of the Bi-2212 whisker composite. The whisker content is $10 \mathrm{wt} \%$.

plate-like shape. Therefore, the closer to the surface, the grain alignment is higher. On the other hand, the grains 
between the whiskers are pressed in the uniaxial direction parallel to the hot pressing direction, because the whiskers are larger than the grains and have the ribbon-like shape (Fig. 3). This uniaxial pressure through the whiskers makes the grains between the whiskers large and highly aligned. This improvement of microstructure by incorporation of the whiskers leads to high $J_{c}$.

$T_{c, \text { zero }}$ and bulk density are $84 \mathrm{~K}$ and $5.8 \mathrm{~g} / \mathrm{cm}^{3}$ for the composite. The relative density for the composite is $89 \%$ of the powder density $\left(6.5 \mathrm{~g} / \mathrm{cm}^{3}\right)$ for the Bi-2212 phase [5]. Although $T_{c, z e r o}$ and density are same with the hot pressed sample without whiskers, $J_{c}$ at $77 \mathrm{~K}$ under zero magnetic field for the composite is $3.1 \times 10^{3} \mathrm{~A} / \mathrm{cm}^{2}$, which is about 8 times higher than the hot pressed sample (Fig. 4). The increase in $J_{c}$ by incorporation of the whiskers is caused by the improvement of microstructure around the whiskers as mentioned above.

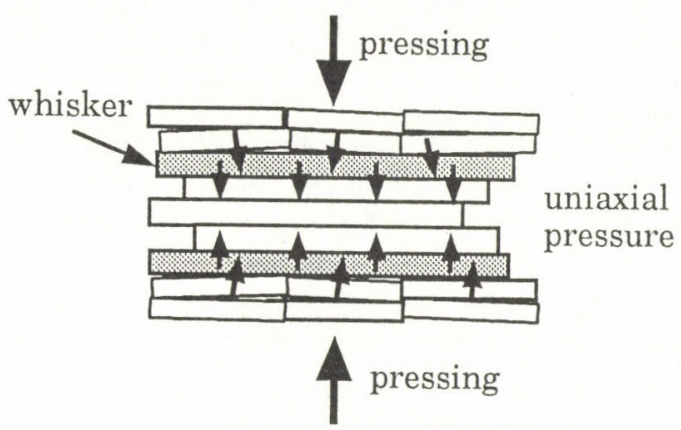

Fig. 3 The improvement of microstructure by incorporation of the whiskers.

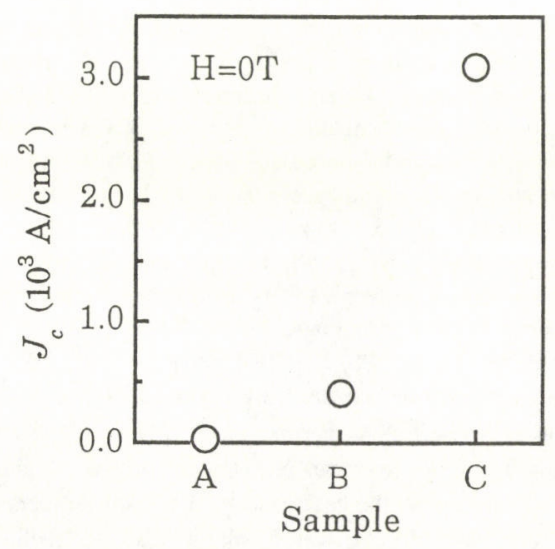

Fig. $4 J_{c}$ for the sintered sample (A), the hot pressed sample (B), and the whisker composite (C) under zero magnetic field. $J_{c}$ was measured for sample $A$ at $69 \mathrm{~K}$, and for sample B and $\mathrm{C}$ at $77 \mathrm{~K}$.

\section{Conclusion}

Hot pressing is effective to hasten the grain growth, align the grains, and improve the bulk density in the Bi-2212 materials. In consequence of hot pressing, $J_{c}$ increases by more than 10 times at $77 \mathrm{~K}$ under zero magnetic field. Hot pressing effect is enhanced by incorporation of large single crystalline Bi-2212 whiskers. $J_{c}$ for the composite is $3.1 \times 10^{3}$ $\mathrm{A} / \mathrm{cm}^{2}$ at $77 \mathrm{~K}$ under zero magnetic field. Although $T_{c, \text { zero }}$ and bulk density are same between the hot pressed sample without the whiskers and the composite, $J_{c}$ for the composite is about 8 times higher than that for the hot pressed sample without the whiskers. This is due to the haste of grain growth and the improvement of grain alignment around the whiskers. These lead to the depression of the effect of grain boundaries on the superconducting current and to increase in $J_{c}$.

\section{References}

1) K. Heine et. al., Appl. Phys. Lett. 55, 2441 (1989).

2) J. Kase et. al., Appl. Phys. Lett. 56, 970 (1990).

3) N. Murayama et. al., Jpn. J. Appl. Phys. 27, L1856 (1988).

4) I. Matsubara et. al., Jpn. J. Appl. Phys. 28, L1121 (1989).

5) S. Nomura et. al., J. Am. Ceram. Soc. 74, 2711 (1991). 